\title{
Link Adaptation in FBMC/OQAM Systems Using NB-LDPC Codes
}

\author{
Màrius Caus*, Monica Navarro*, Xavier Mestre* and Ana Pérez-Neira*† \\ ${ }^{*}$ Centre Tecnològic de Telecomunicacions de Catalunya (CTTC), Castelldefels, Barcelona, Spain \\ ${ }^{\dagger}$ Dept. of Signal Theory and Communications, Universitat Politècnica de Catalunya (UPC), Barcelona, Spain
}

\begin{abstract}
This paper investigates the application of nonbinary low density parity check (NB-LDPC) codes to offset-QAM based filter bank multicarrier systems, known as FBMC/OQAM. The analysis conducted in the paper reveals that the mapping scheme can be optimized to simplify detection and minimize the correlation of the noise. Based on the proposed modifications, existing adaptive coding and modulation (ACM) algorithms can be easily tailored to accommodate the characteristics of NBLDPC and FBMC/OQAM. In this paper, priority has been given to mutual information-based ACM algorithms. Numerical results verify that the proposed algorithm guarantees the target block error rate.
\end{abstract}

\section{INTRODUCTION}

The future of mobile communication networks towards the so-called 5G networks may result either as an evolution or a revolution of current long-term evolution (LTE) systems. Although it is difficult to foresee the next evolutionary step, it is clear that the air interface will need to cope with a multi-service environment and heterogeneity of network components. Ongoing research projects, like FANTASTIC-5G [1], consider the design of a highly flexible, versatile and scalable air interface to cope with challenging requirements in terms of data rate, latency, reliability and energy consumption. This motivates the adoption of air interfaces that achieve a finegrained control of the spectrum, such as filter bank multicarrier (FBMC) systems, instead of orthogonal frequency division multiplexing (OFDM). In this regard, the FBMC scheme combined with the offset quadrature amplitude modulation, referred to as FBMC/OQAM or OFDM/OQAM [2], has emerged as an eligible candidate [3]. The most appealing features of FBMC/OQAM are the reduced out-of-band radiation and the absence of cyclic prefix (CP).

To meet the throughput and the reliability requirements, the use of link adaptation algorithms with adaptive coding and modulation (ACM) becomes crucial. Consequently, a question arises on whether current solutions will be valid for envisioned new air interfaces. Therefore, attention must be paid to the features that the future air interface might incorporate: the relaxation of the orthogonality properties and the integration of multiple antenna schemes with new waveform designs. The former addresses the analysis of non-orthogonal effects on the link adaptation algorithm and incorporates a new metric to account for it. The second motivates the adoption of nonbinary low density parity check (NB-LDPC) codes as the constituent forward error correcting (FEC) scheme. NB-LDPC codes are known to outperform the binary counterpart for low to moderate code lengths [4], [5]. In multi-antenna transmission systems, performance differences can be significantly higher, because non-binary codes have shown an inherent advantage [6], [7]. Furthermore, NB-LDPC enables a simple repetition coding scheme suitable for integration of ACM with hybrid automatic repeat request (HARQ) retransmissions, which provides additional gain to binary coding repetition and soft combining [8], [9].

In the following sections, it will be shown that the combination of the aforementioned techniques is not trivial, requiring specific channel quality metrics and mapping schemes to efficiently transmit the code symbols. More specifically, in this paper we establish the framework for the general case, starting with the analysis of the proposed ACM for FBMC/OQAM and NB-LDPC for the single antenna case. We leverage on the algorithm presented in [10], which showed excellent complexity-performance trade-off under OFDM transmissions, to further develop it for non-binary processing and establish a suitable metric accounting for the characteristics of the FBMC/OQAM modulation.

The remaining of this paper is organized as follows. Section II reviews the basics of NB-LDPC codes and provides the reference performance curves for rate selection. The system model is introduced in Section III. Section IV describes the application of mutual information-based ACM algorithms for FBMC/OQAM. Finally, numerical results are presented in Section V and the conclusions are drawn in Section VI.

\section{NB-LDPC CODES}

This section provides the reference performance curves as function of the mutual information, in order to optimize the rate selection subject to block error rate (BLER) constraints. The reference curves are obtained from simulations in additive white Gaussian noise (AWGN) channels. Next, we introduce the notation and specify the expressions for the mutual information.

Like its binary counterpart, the non-binary LDPC code is defined as the set of codewords $\mathbf{c}=\left[c_{1}, \cdots, c_{N}\right]^{T}$ that satisfy the parity-check equation $\mathbf{H c}=\mathbf{0}$, where $\mathbf{H} \in$ $\mathbb{F}_{q}^{N-K \times N}$ is the parity-check matrix defined over the Galois field $\mathbb{F}_{q}=\{0,1, \cdots, q-1\}$. Let $K$ be the length of the information block and $q$ the field order, which is a multiple of 2. Denoting the generator matrix by $\mathbf{G} \in \mathbb{F}_{q}^{N \times K}$, each codeword is generated according to $\mathbf{c}=\mathbf{G u}$, with $\mathbf{u}=$ $\left[u_{1}, \cdots, u_{K}\right]^{T} \in \mathbb{F}_{q}^{K \times 1}$ being the input data sequence or block 


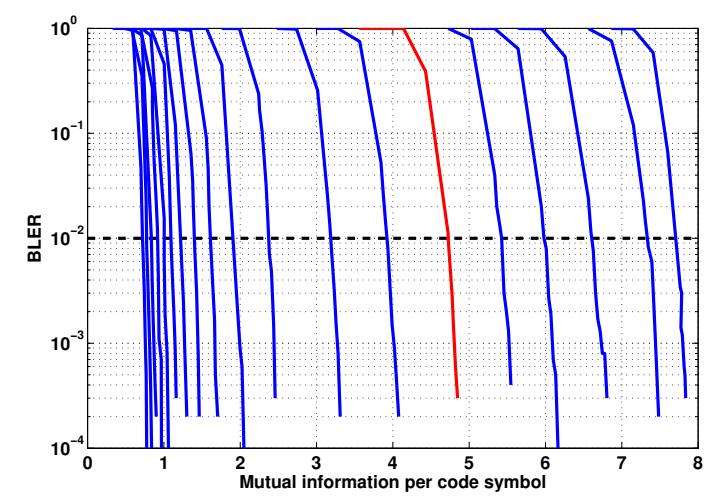

Figure 1. BLER against the mutual information per code symbol with 256QAM over an AWGN channel.

message. Then, code symbols are mapped into a $T$ complex dimensional vector from the set $\mathcal{X}=\left\{\mathbf{a}_{0}, \cdots, \mathbf{a}_{q-1}\right\}$, where $\mathbf{a}_{i} \in \mathbb{C}^{T \times 1}$. The multidimensional constellation $\mathcal{X}$ can be built from the Cartesian product of the standard $M_{s}$-QAM constellations with $q=\left(M_{s}\right)^{T}$ or from sphere packings [7]. In general, they will depend on the mapping to the number of transmit antennas and channel uses. For the single antenna case, which is the case tackled in this paper, the code symbols are transmitted into $T$ consecutive time slots and $\mathcal{X}$ is built from the Cartesian product of $M_{s}$-QAM constellations. For the ease of exposition, the labeling is compactly expressed as $\boldsymbol{\mu}\left(c_{l}\right)=\mathbf{x}_{l}=\left[x_{l, 1}, \cdots, x_{l, T}\right]^{T}$, for $1 \leq l \leq N$, where $c_{l} \in \mathbb{F}_{q}$ and $\mathbf{x}_{l} \in \mathcal{X}$. It is assumed that $\mathbb{E}\left\{\left|x_{l, i}\right|^{2}\right\}=E_{S}$, for $1 \leq i \leq T$.

The received vector is given by $\mathbf{y}_{l}=\mathbf{x}_{l}+\mathbf{w}_{l}$, with $\mathbf{w}_{l} \sim \mathcal{C N}\left(\mathbf{0}, N_{0} \mathbf{I}_{T}\right)$ and $\mathbf{I}_{T}$ being the $T$-dimensional identity matrix. The receiver computes the soft information loglikelihoods by demapping the vector $\mathbf{y}_{l}$ into the L-vector $\mathbf{L}_{l}=\left[\mathrm{L}_{l, 0}, \mathrm{~L}_{l, 1}, \cdots, \mathrm{L}_{l, q-1}\right]^{T}$, whose $i$ th element is [7]:

$$
\mathrm{L}_{l, i}=-\frac{1}{N_{0}}\left(\left\|\mathbf{y}_{l}-\mathbf{a}_{i}\right\|_{2}^{2}-\left\|\mathbf{y}_{l}-\mathbf{a}_{0}\right\|_{2}^{2}\right), 0 \leq i \leq q-1 .
$$

We consider the implementation in [11] for the NB-LDPC decoder. Concerning the code, it is proposed to leverage on ultra-sparse NB-LPDC codes [4]. The field order has been set to $q=256$ and the message length is $K=90$. Building upon the mother code of rate $1 / 2$, a wide range of code rates can be obtained by puncturing and non-binary repetition. More specifically, the code rates $R \triangleq K / N$ available for link adaptation are

$$
\begin{aligned}
R= & \left\{\frac{1}{14}, \frac{1}{13}, \frac{1}{12}, \frac{1}{11}, \frac{1}{10}, \frac{1}{9}, \frac{1}{8}, \frac{1}{7}, \frac{1}{6}, \frac{1}{5}, \frac{1}{4}, \frac{1}{3}, \frac{9}{22},\right. \\
& \left.\frac{1}{2}, \frac{3}{5}, \frac{2}{3}, \frac{3}{4}, \frac{5}{6}, \frac{9}{10}\right\} .
\end{aligned}
$$

Mapping and demapping simplifies significantly if exactly one code symbol is mapped into a vector of QAM symbols. Hence, code symbols that belong to $\mathbb{F}_{256}$, can be mapped into 256QAM and 16-QAM constellations, with $T=1$ and $T=2$, respectively. With symbols drawn from the $2^{m}$-QAM alphabet $\mathcal{X}_{m}=\left\{s_{1}, \cdots, s_{2^{m}}\right\}$, the mutual information per code symbol

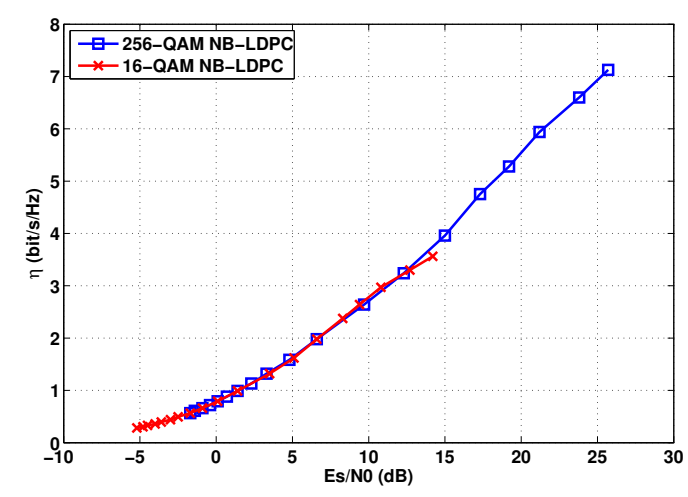

Figure 2. Throughput against $\frac{E_{S}}{N_{0}}$ over an AWGN channel when $\mathrm{BLER}=10^{-2}$.

is defined with this closed-form expression [6]:

$$
\begin{aligned}
& I\left(\frac{E_{S}}{N_{0}}\right)=\frac{\log _{2}(256)}{m}\left(m-\frac{1}{2^{m}}\right. \\
& \left.\times \sum_{j=1}^{2^{m}} \mathbb{E}\left\{\log _{2}\left(\sum_{i=1}^{2^{m}} e^{-\left|\sqrt{\frac{E_{S}}{N_{0}}}\left(s_{j}-s_{i}\right)+w\right|^{2}+|w|^{2}}\right)\right\}\right),
\end{aligned}
$$

where $w$ is a circular symmetric Gaussian variable distributed as follows: $w \sim \mathcal{C N}(0,1)$. It has been assumed that symbols are equi-probable with a mean energy equal to 1 , i.e. $\frac{1}{2^{m}} \sum_{i}\left|s_{i}\right|^{2}=1$. The performance of the code is shown in Figure 1, which represents the BLER against the mutual information per code symbol with 256-QAM. Due to space limitations the curves for 16-QAM are not depicted. The red curve corresponds to the mother code performance. For selecting the modulation and coding scheme (MCS) it is useful to represent the (throughput, $\frac{E_{S}}{N_{0}}$ ) pairs as in Figure 2. The $\frac{E_{S}}{N_{0}}$ threshold is obtained by inverting (3) for the mutual information threshold at the target BLER (e.g. BLER $=10^{-2}$ ) from Figure 1. The modulation and the code rate are mapped to the throughput value as follows:

$$
\eta=\frac{\log _{2}(256)}{T} \frac{K}{N}(1-\text { BLER }) .
$$

Note that for this particular scenario, the throughput curves of 256-QAM and 16-QAM overlap for $-1.7 \leq \frac{E_{S}}{N_{0}} \leq 15$. In this range, we propose to favor 256-QAM to the detriment of 16-QAM. The reason lies in the fact that the mapping scheme is simplified when 256-QAM is employed, because one code symbol is transmitted in one channel access. With 16-QAM two accesses are required. This choice will have an impact on the link adaptation algorithm as detailed in Section IV.

\section{SYSTEM MODEL}

This section presents the system model when the air interface is based on the FBMC/OQAM modulation scheme [2]. It is pertinent to stress that FBMC/OQAM exhibits a reduced out-of-band radiation, thanks to good spectral confinement of the synthesis filters

$$
f_{m}[n]=p[n] e^{j \frac{2 \pi}{M} m\left(n-\frac{L_{p}-1}{2}\right)}, \quad 0 \leq n \leq L_{p}-1,
$$


where $p[n]$ is the prototype pulse, the length of which is $L_{p}$. When the band is partitioned into $M$ subcarriers, the inputoutput relation at the $q$ th subcarrier is given by [12]:

$$
y_{q}[k]=\sum_{m=q-1}^{q+1} g_{q m}[k] \star\left(\sqrt{E_{S}} \theta_{m}[k] d_{m}[k]\right)+w_{q}[k],
$$

for $0 \leq q \leq M-1$. Owing to the excellent frequency localization properties of $p[n]$, it is common to assume that only adjacent subcarriers overlap. It is worth recalling that the FBMC/OQAM transmission format hinges on introducing a time offset, which is equal to half the symbol period, between real and imaginary parts of the QAM symbols [2]. As a result, it is more convenient to express the demodulated signal as function of the PAM modulated symbols $\left\{d_{m}[k]\right\}$, which have a mean energy equal to $1 / 2$. To complete the characterization of the transmitted data, the phase term is defined as

$$
\theta_{m}[k]= \begin{cases}1 & m+k \text { even } \\ j & m+k \text { odd }\end{cases}
$$

to guarantee that symbols transmitted in contiguous timefrequency $(\mathrm{TF})$ positions have a difference of phase equal to $\frac{\pi}{2}$. In presence of multipath fading, the equivalent channel between the $m$ th and the $q$ th subcarrier and the filtered noise are formulated as follows:

$$
\begin{gathered}
g_{q m}[k]=\sum_{l} h[l] \sum_{n} f_{m}[n] f_{q}^{*}\left[n+l-\frac{M}{2} k\right] \\
w_{q}[k]=\sum_{n} w[n] f_{q}^{*}\left[n-\frac{M}{2} k\right] .
\end{gathered}
$$

The impulse response of the channel and the noise are denoted by $h[n]$ and $w[n]$, respectively. When the channel frequency response is flat in a small neighborhood around the TF position $(k, q),(8)$ is approximated by

$$
g_{q m}[k] \approx H(q) \sum_{n} f_{m}[n] f_{q}^{*}\left[n-\frac{M}{2} k\right],
$$

where $H(q)$ denotes the discrete-time Fourier transform (DTFT) of $h[n]$ evaluated at $\frac{2 \pi}{M} q$. In this case, provided that the perfect reconstruction (PR) property is satisfied, namely

$\Re\left(\sum_{n} f_{m}\left[n-\frac{M}{2} l\right] \theta_{m}[l] f_{q}^{*}\left[n-\frac{M}{2} k\right] \theta_{q}^{*}[k]\right)=\delta_{q, m} \delta_{k, l}$,

interference can be easily removed by resorting to single-tap equalization. Then, the estimated symbols can be written as

$$
\begin{aligned}
\hat{d}_{q}[k] & =\Re\left(\frac{\theta_{q}^{*}[k] y_{q}[k]}{H(q)}\right) \\
& =\sqrt{E_{S}} d_{q}[k]+i_{q}[k]+\Re\left(\frac{\theta_{q}^{*}[k] w_{q}[k]}{H(q)}\right) .
\end{aligned}
$$

If (10) holds true $i_{q}[k]=0$. Otherwise, the channel is not perfectly equalized and, consequently, the residual error is not negligible.

The rest of the section is devoted to tailoring the scheme presented in Section II to the lattice structure of FBMC/OQAM. First, it is important to remark that PAM symbols can be obtained from real and imaginary parts of complex-valued QAM symbols. Hence, the elements of the coded sequence c can be straightforwardly mapped into $2 T$ TF positions, depending on the constellation of choice. The question that remains open is how to select the $2 T$ contiguous TF positions, so that the noise is uncorrelated. To cast some light into this problem, lets express the noise as follows:

$$
\begin{aligned}
\eta_{q}[k]= & \Re\left(\frac{\theta_{q}^{*}[k] w_{q}[k]}{H(q)}\right)=\Re\left(\frac{1}{H(q)}\right) \Re\left(\theta_{q}^{*}[k] w_{q}[k]\right) \\
& -\Im\left(\frac{1}{H(q)}\right) \Im\left(\theta_{q}^{*}[k] w_{q}[k]\right) .
\end{aligned}
$$

To determine $\mathbb{E}\left\{\eta_{q}[k] \eta_{m}[l]\right\}$, we rely on these correlations:

$$
\begin{aligned}
& \mathbb{E}\left\{\Re\left(\theta_{m}^{*}[l] w_{m}[l]\right) \Re\left(\theta_{q}^{*}[k] w_{q}[k]\right)\right\}=\frac{N_{0}}{2} \\
& \times \sum_{n} \Re\left(f_{m}\left[n-\frac{M}{2} l\right] \theta_{m}[l] f_{q}^{*}\left[n-\frac{M}{2} k\right] \theta_{q}^{*}[k]\right) \\
& =\mathbb{E}\left\{\Im\left(\theta_{q}^{*}[k] w_{q}[k]\right) \Im\left(\theta_{m}^{*}[l] w_{m}[l]\right)\right\} \\
& \mathbb{E}\left\{\Re\left(\theta_{m}^{*}[l] w_{m}[l]\right) \Im\left(\theta_{q}^{*}[k] w_{q}[k]\right)\right\}=\frac{N_{0}}{2} \\
& \times \sum_{n} \Im\left(f_{m}\left[n-\frac{M}{2} l\right] \theta_{m}[l] f_{q}^{*}\left[n-\frac{M}{2} k\right] \theta_{q}^{*}[k]\right) \\
& =-\mathbb{E}\left\{\Re\left(\theta_{q}^{*}[k] w_{q}[k]\right) \Im\left(\theta_{m}^{*}[l] w_{m}[l]\right)\right\} .
\end{aligned}
$$

The equalities have been obtained under the assumption that the noise samples satisfy $w[n] \sim \mathcal{C N}\left(0, N_{0}\right)$. Taking into account (14),(15) along with (11), the noise correlation becomes

$$
\begin{aligned}
& \mathbb{E}\left\{\eta_{q}[k] \eta_{m}[l]\right\}=\delta_{q, m} \delta_{k, l} \frac{N_{0}}{2} \Re\left(\frac{1}{H(q)^{*} H(m)}\right) \\
& +\frac{N_{0}}{2} \Im\left(\frac{1}{H(q)^{*} H(m)}\right) \\
& \times \sum_{n} \Im\left(f_{m}\left[n-\frac{M}{2} l\right] \theta_{m}[l] f_{q}^{*}\left[n-\frac{M}{2} k\right] \theta_{q}^{*}[k]\right) .
\end{aligned}
$$

In the light of this result, it can be resolved that the best strategy to map the code symbols is to keep fixed the subcarrier index. Then, it follows that the correlation matrix of $\boldsymbol{\eta}_{q}[k]=$ $\left[\eta_{q}[k] \cdots \eta_{q}[k+2 T-1]\right]^{T}$ is diagonal, i.e.,

$$
\mathbb{E}\left\{\boldsymbol{\eta}_{q}[k] \boldsymbol{\eta}_{q}^{T}[k]\right\}=\frac{N_{0}}{2|H(q)|^{2}} \mathbf{I}_{2 T} .
$$

In this case the QAM symbols can be easily retrieved by processing two consecutive time slots as follows:

$$
\begin{aligned}
\bar{y}_{q}\left[k^{\prime}\right] & =\hat{d}_{q}\left[2 k^{\prime}\right]+j \hat{d}_{q}\left[2 k^{\prime}+1\right] \\
& =\sqrt{E_{S}} \bar{x}_{q}\left[k^{\prime}\right]+\bar{i}_{q}\left[k^{\prime}\right]+\bar{\eta}_{q}\left[k^{\prime}\right],
\end{aligned}
$$

with $k^{\prime}=\frac{k}{2}$, due to the rate conversion. For $0 \leq k \leq N$, the sampling index $k^{\prime}$ fulfills $0 \leq k^{\prime} \leq \frac{N}{2}$. From (18) it can be inferred that $\bar{x}_{q}\left[k^{\prime}\right]$ is either 16- or 256-QAM modulated with a mean energy equal to 1 , the variance of $\bar{\eta}_{q}\left[k^{\prime}\right]$ is $N_{0} /|H(q)|^{2}$ and the power of the residual error is [13]:

$$
\sigma_{q}^{2}=\mathbb{E}\left\{\left|\bar{i}_{q}\left[k^{\prime}\right]\right|^{2}\right\}=\frac{E_{S}}{4(M / 2)^{3}} \frac{\left|H(q)^{\prime}\right|^{2}}{|H(q)|^{2}} C_{p} .
$$

The constant $C_{p}$ depends on the prototype pulse and $H(q)^{\prime}$ accounts for the derivative of the DTFT of $h[n]$ evaluated at 
$\frac{2 \pi}{M} q$. Therefore, the quality metric associated with $\bar{x}_{q}\left[k^{\prime}\right]$ is

$$
\gamma_{q}=\frac{E_{S}}{\frac{N_{0}}{|H(q)|^{2}}+\sigma_{q}^{2}} .
$$

At this point, it can be established that these TF positions $\{(k, q), \cdots,(k+2 T-1, q)\}$ form the best selection to minimize the correlation of the noise. Besides, this selection paves the way to computing the L-vector. To prove it, imagine that the OQAM post-processing of $2 T$ samples gives rise to $\left\{\bar{y}_{q}\left[k^{\prime}\right], \bar{y}_{q}\left[k^{\prime}+T-1\right]\right\}$. If the QAM symbols $\left\{\bar{x}_{q}\left[k^{\prime}\right], \bar{x}_{q}\left[k^{\prime}+T-1\right]\right\}$ convey information of the code symbol $c_{l} \in \mathbb{F}_{256}$, then the demaper can straightforwardly compute the 256 symbol-levels as follows:

$\mathrm{L}_{l, i}=\sum_{j=0}^{T-1} \frac{\left|\bar{y}_{q}\left[k^{\prime}+j\right]-\sqrt{E_{S}} s_{0, j}\right|^{2}}{\frac{N_{0}}{|H(q)|^{2}}+\sigma_{q}^{2}}-\frac{\left|\bar{y}_{q}\left[k^{\prime}+j\right]-\sqrt{E_{S}} s_{i, j}\right|^{2}}{\frac{N_{0}}{|H(q)|^{2}}+\sigma_{q}^{2}}$,

for $i=0, \cdots, 255$. For $T=1 s_{i, 0} \in 256$-QAM, while for $T=2 s_{i, 0}, s_{i, 1} \in 16$-QAM.

\section{LINK ADAPTATION}

This section details the link adaptation solution. Special attention is given to implementation aspects that arise from the application of NB-LDPC and FBMC/OQAM in frequency selective channels. Suppose that the scheduler assigns $L$ RBs to a given user, which may not be contiguous. The triplet $\left(m(l), p_{l}, \beta_{l}\right)$ denotes the number of bits per symbol, the transmit power and the signal to interference plus noise ratio (SINR) associated with the $l$ th RB. Depending on the RB size and the propagation conditions, the fading may not be flat within one RB. It is assumed that the RB is formed by $n_{f}$ subcarriers and $n_{t}$ time slots. The SINR measured in all the $\mathrm{TF}$ positions can be mapped into this single metric [10]:

$$
\beta_{l}=\alpha f^{-1}\left(\frac{1}{n_{t} n_{f}} \sum_{i=1}^{n_{f}} \sum_{j=1}^{n_{t}} f\left(\gamma_{i j}^{l}\right)\right)+(1-\alpha) \min _{i, j}\left(\gamma_{i j}^{l}\right) .
$$

where $f(x)=\log _{2}(1+x)$ and $\gamma_{i j}^{l}$ is the SINR measured in the $(i, j)$-th TF position of the $l$-th RB, which is computed in (20). As [10] suggests we have set $\alpha=0.85$. The ACM algorithm introduced in [10] relies on the mutual information. Under the assumption that $\beta_{1} \leq \cdots \leq \beta_{L}$ and that the transmit energy is $E_{T}=n_{f} E_{S} L$, the algorithm guarantees the BLER to be below a target threshold by executing the following steps.

1) Distribute the power uniformly, i.e. $p_{l}=1 \forall l$.

2) Select the MCS on a per RB basis $(1 \leq l \leq L)$. According to (2), there are 38 MCSs. However, from Figure 2 the set of MCS can be reduced to 27 by switching the modulation according to the following criterion

$$
\begin{aligned}
& 10 \log _{10}\left(p_{l} \beta_{l}\right) \geq-1.7 \rightarrow m(l)=8 \quad \text { (256-QAM) } \\
& 10 \log _{10}\left(p_{l} \beta_{l}\right)<-1.7 \rightarrow m(l)=4 \quad \text { (16-QAM). }
\end{aligned}
$$

As Table I indicates, 16-QAM can only be used with a reduced number of code rates. If $10 \log _{10}\left(p_{l} \beta_{l}\right)<-5.2$, the $l$ th RB is discarded, because the constraint BLER $\leq 10^{-2}$ is violated. Consequently, the energy assigned to the lth RB
Table I

MODULATION AND CODING SCHEMES

\begin{tabular}{|c|c|c|c|c|c|}
\hline Index & MCS & Index & MCS & Index & MCS \\
\hline 0 & $\left(16, \frac{1}{14}\right)$ & 9 & $\left(256, \frac{1}{13}\right)$ & 18 & $\left(256, \frac{1}{4}\right)$ \\
\hline 1 & $\left(16, \frac{1}{13}\right)$ & 10 & $\left(256, \frac{1}{12}\right)$ & 19 & $\left(256, \frac{1}{3}\right)$ \\
\hline 2 & $\left(16, \frac{1}{12}\right)$ & 11 & $\left(256, \frac{1}{11}\right)$ & 20 & $\left(256, \frac{9}{22}\right)$ \\
\hline 3 & $\left(16, \frac{1}{11}\right)$ & 12 & $\left(256, \frac{1}{10}\right)$ & 21 & $\left(256, \frac{1}{2}\right)$ \\
\hline 4 & $\left(16, \frac{1}{10}\right)$ & 13 & $\left(256, \frac{1}{9}\right)$ & 22 & $\left(256, \frac{3}{5}\right)$ \\
\hline 5 & $\left(16, \frac{1}{9}\right)$ & 14 & $\left(256, \frac{1}{8}\right)$ & 23 & $\left(256, \frac{2}{3}\right)$ \\
\hline 6 & $\left(16, \frac{1}{8}\right)$ & 15 & $\left(256, \frac{1}{7}\right)$ & 24 & $\left(256, \frac{3}{4}\right)$ \\
\hline 7 & $\left(16, \frac{1}{7}\right)$ & 16 & $\left(256, \frac{1}{6}\right)$ & 25 & $\left(256, \frac{5}{6}\right)$ \\
\hline 8 & $\left(256, \frac{1}{14}\right)$ & 17 & $\left(256, \frac{1}{5}\right)$ & 26 & $\left(256, \frac{9}{10}\right)$ \\
\hline
\end{tabular}

per time slot, i.e. $n_{f} E_{S}$, is uniformly redistributed among the rest of RBs. Therefore, $p_{l}>1$ if the number of active RBs is lower than $L$. Once the energy is assigned and the modulation is chosen, the code rate is determined from the BLER performance curves (Figure 1) and the evaluation of $I\left(p_{l} \beta_{l}\right)$ in (3). We shall remark that the on-line computation of (3) is unaffordable. However, the mutual information can be efficiently computed by linear interpolation, provided that some values are computed beforehand.

3) Denoting by $N_{l}$ the codeword length assigned to the $l$ th $\mathrm{RB}$, the code rate that is applied to all RBs is computed as

$$
R_{\mathrm{all}}=\frac{\sum_{l \in \mathbb{S}_{a}} m(l) \frac{K}{N_{l}}}{\sum_{l \in \mathbb{S}_{a}} m(l)} .
$$

Note that the set $\mathbb{S}_{a}$, the cardinality of which is $L_{a}$, gathers the index of the active RBs. Hence, $N_{C S}=n_{t} n_{f} \sum_{l \in \mathbb{S}_{a}} \frac{m(l)}{\log _{2}(256)}$ is the number of transmitted code symbols.

4) Determine the number of codewords as $N_{C W}=$ $\left\lfloor N_{C S} R_{\text {all }} / K\right\rfloor$ and calculate the codeword length as $N_{\text {all }}=$ $\left\lfloor N_{C S} / N_{C W}\right\rfloor$. Clearly $\frac{K}{N_{\text {all }}}$ may not coincide with the exact rate values in (2). If so, the codeword length selection is based on the average mutual information. More specifically, compute first the average mutual information per code symbol as

$$
\bar{I}=\frac{\sum_{l \in \mathbb{S}_{a}} m(l) I\left(p_{l} \beta_{l}\right)}{\sum_{l \in \mathbb{S}_{a}} m(l)} .
$$

Then, select the closest rate that fulfills the requirement. To this end we shall find the two contiguous codeword lengths $\left(N_{l b}, N_{u b}\right)$ from (2) that satisfy $N_{l b} \leq N_{\text {all }} \leq N_{u b}$. If the mutual information threshold $I_{l b}$ associated to code rate $\frac{K}{N_{l b}}$ (obtained from performance curves Figure 1 ) is above the average mutual information, i.e. $I_{l b}>\bar{I}$, then $N_{\text {all }}=N_{l b}$. Otherwise, the codeword length is set to $N_{\text {all }}=N_{u b}$, which results in a more conservative choice than the exact value computed by the algorithm. Finally, the number of codewords has to be updated as $N_{C W}=\left\lfloor N_{C S} / N_{\text {all }}\right\rfloor$.

5) Interleave the $N_{C W}$ codewords, and assign them to the $L_{a}$ active RBs. Then, feed the constellation symbols into the FBMC/OQAM modulator.

\section{NumericAl RESUlts}

This section presents simulations-based results to assess the algorithm described in Section IV. The FBMC/OQAM 


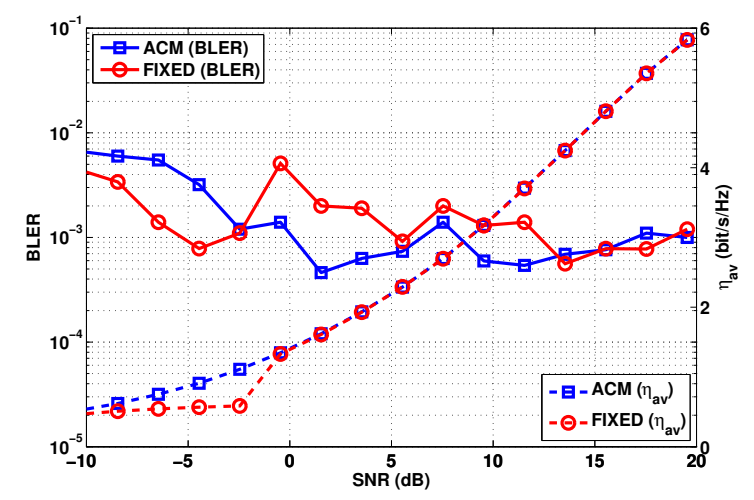

Figure 3. Throughput vs. SNR and BLER vs. SNR.

prototype pulse $p[n]$ is designed according to the frequency sampling approach proposed in [14] with an overlapping factor equal to 4 . The rest of parameters are based on the LTE numerology, i.e. only $M_{a}=600$ out of $M=1024$ subcarriers are active and the sampling frequency is $f_{s}=15.36 \mathrm{MHz}$. The available time/frequency resources are arranged in 50 RBs. Each RB consists of $n_{f}=12$ subcarriers and $n_{t}=14$ time slots. In each time slot two PAM modulated symbols are received, which is equivalent to saying that one QAM symbol can be retrieved per time slot. As for the propagation conditions, the channel obeys the extended pedestrian A model [15].

For the assessment we evaluate the throughput when the ACM algorithm is implemented targeting a BLER $=10^{-2}$. In Figure 3 the curves are represented over the signal to noise ratio (SNR), namely

$$
\mathrm{SNR}=\frac{R_{S} E_{T}}{N_{0} B}
$$

where the symbol rate is $R_{S}=f_{s} / M, E_{T}$ accounts for the multicarrier symbol energy, $N_{0}$ is the noise spectral density and the transmission bandwidth is denoted by $B=10 \mathrm{MHz}$. The average throughput for a given channel realization is given by

$$
\eta_{a v}=\frac{K}{N_{\mathrm{all}}}(1-\mathrm{BLER}) \frac{1}{L_{a}} \sum_{l \in \mathbb{S}_{a}} m(l) .
$$

In Figure $3 \mathrm{ACM}$ is compared to an algorithm that fixes the modulation across RBs. For a fair comparison, the benchmark can only select the MCSs gathered in Table I. Figure 3 confirms that significant throughput gains are observed at low SNR when ACM is implemented.

\section{CONCLUSiOnS}

The work presented in this paper delves into the application of ACM for next generation wireless communication systems, where FBMC/OQAM and NB-LDPC may play a central role. The implementation aspects of these two key technology components have been investigated, leading to specific channel quality metrics and mapping schemes. The proposed modifications simplify detection, minimize the correlation of the noise and allow existing ACM algorithms to be successfully applied. It has been experimentally verified that competitive results can be achieved with respect to ACM, which provides the highest throughput, by fixing the modulation. This conclusion may not be valid for MIMO configurations and different code rate sets, highlighting that further research is needed in other scenarios.

\section{ACKNOWLEDGMENT}

Part of this work has been performed in the framework of the Horizon 2020 project FANTASTIC-5G (ICT-671660), which is partly funded by the European Union. The authors would like to acknowledge the contributions of their colleagues in FANTASTIC$5 \mathrm{G}$. This work has also received funding from the Spanish Government under projects TEC2014-59255-C3-1-R and Red ARCO $5 \mathrm{G}$ TEC2014-56469-REDT and from the Catalan Government under grant 2014SGR1567.

\section{REFERENCES}

[1] "Flexible Air iNTerfAce for Scalable service delivery wiThin wIreless Communication networks of the 5th Generation (FANTASTIC 5G)." [Online]. Available: http://fantastic5g.eu/

[2] P. Siohan, C. Siclet, and N. Lacaille, "Analysis and design of OFDM/OQAM systems based on filterbank theory," IEEE Trans. Signal Process., vol. 50, no. 5, pp. 1170-1183, May 2002.

[3] P. Banelli, S. Buzzi, G. Colavolpe, A. Modenini, F. Rusek, and A. Ugolini, "Modulation Formats and Waveforms for 5G Networks: Who Will Be the Heir of OFDM?: An overview of alternative modulation schemes for improved spectral efficiency," IEEE Signal Process. Mag., vol. 31, no. 6, pp. 80-93, Nov 2014.

[4] C. Poulliat, M. Fossorier, and D. Declercq, "Design of regular (2,dc)LDPC codes over GF(q) using their binary images," IEEE Trans. Commun., vol. 56, no. 10, pp. 1626-1635, October 2008.

[5] G. Liva, E. Paolini, T. De Cola, and M. Chiani, "Codes on high-order fields for the CCSDS next generation uplink," in Advanced Satellite Multimedia Systems Conference (ASMS) and 12th Signal Processing for Space Communications Workshop (SPSC), Sept 2012, pp. 44-48.

[6] S. Pfletschinger and D. Declercq, "Getting Closer to MIMO Capacity with Non-Binary Codes and Spatial Multiplexing," in Global Telecommunications Conference (GLOBECOM), Dec 2010, pp. 1-5.

[7] _ _ "Non-binary coding for vector channels," in IEEE 12th International Workshop on Signal Processing Advances in Wireless Communications (SPAWC), June 2011, pp. 26-30.

[8] K. Kasai, D. Declercq, and K. Sakaniwa, "Fountain Coding via Multiplicatively Repeated Non-Binary LDPC Codes," IEEE Trans. Commun., vol. 60, no. 8, pp. 2077-2083, August 2012.

[9] S. Pfletschinger, D. Declercq, and M. Navarro, "Adaptive HARQ With Non-Binary Repetition Coding," IEEE Trans. Wireless Commun., vol. 13, no. 8, pp. 4193-4204, Aug 2014.

[10] S. Stiglmayr, M. Bossert, and E. Costa, "Adaptive Coding and Modulation in OFDM Systems using BICM and Rate-Compatible Punctured Codes," in European Wireless, 2007, pp. 102-107.

[11] L. Barnault and D. Declercq, "Fast decoding algorithm for LDPC over GF(2q)," in IEEE Information Theory Workshop, March 2003, pp. 7073.

[12] M. Caus, A. I. Perez Neira, and M. Renfors, "Low-complexity interference variance estimation methods for coded multicarrier systems: application to SFN," EURASIP Journal on Advances in Signal Processing, no. 1, pp. 1-11, 2013.

[13] X. Mestre, M. Majoral, and S. Pfletschinger, "An asymptotic approach to parallel equalization of filter bank based multicarrier signals," IEEE Trans. Signal Process., vol. 61, no. 14, pp. 3592-3606, July 2013.

[14] M. Bellanger, "Specification and design of a prototype filter for filter bank based multicarrier transmission." ICASSP, 2001, pp. 2417-2420.

[15] "3rd Generation Partnership Project; Technical Specification Group Radio Access Network; Evolved Universal Terrestrial Radio Access (E-UTRA); User Equipment (UE) radio transmission and reception; (Release 8).” 3GPP TR 36.803v1.1.0. 